\title{
一种氟代腺苷类衍生物的合理设计与合成
}

\author{
许芝祥 尹伟陈晶磊乔春华* \\ (苏州大学药学院 苏州 215123)
}

\begin{abstract}
摘要 针对抗结核药物新靶酶一一腺苷酰化酶家族的泛酸合成酶，以生物电子等排原理，模拟泛酸合成酶催化的中间 体，用氟取代中间体糖环上 $2^{\prime}$ 位羟基，合理设计了目标化合物 5'- $O$-\{[ $(R)-2$-羟基-3,3-二甲基丁酰基]磺酰胺基\}-2'-去氧$2^{\prime}$-氟腺苷(1). 以 $D$-叔亮氨酸和阿糖腺苷为原料, 通过 9 步反应合成得到目标产物. 其结构经 ${ }^{1} \mathrm{H} ~ \mathrm{NMR},{ }^{13} \mathrm{C} \mathrm{NMR}$ 和 HRMS 表征确认.

关键词 氟代腺苷衍生物; 泛酸合成酶; 抗结核; 全合成
\end{abstract}

\section{Rational Design and Synthesis of a Fluorinated Adenosine Derivative}

\author{
$\mathrm{Xu}$, Zhixiang Yin, Wei Chen, Jinglei Qiao, Chunhua* \\ (College of Pharmacy, Soochow University, Suzhou 215123)
}

\begin{abstract}
Pantothenate synthetase (PS), a member of adenosine acylation enzyme family, was the new target of anti-tuberculosis drug development. Employing bioisostere principle, 5'- $O$ - $\{[(R)$-2-hydroxy-3,3-dimethylbutanoyl $]$ sulfamoyl $\}$ 2'-deoxy-2'-fluoroadenosine (1), a mimic of the PS catalyzed reaction intermediate, was rational designed by substituting the 2'-hydroxyl group of intermediate sugar ring with fluorine. This compound was synthesized using $D$-tertiary leucine and vidarabine as the starting materials by 9 steps of reaction, and its structure was fully characterized by ${ }^{1} \mathrm{H} N M R,{ }^{13} \mathrm{C}$ NMR and HR-MS techniques.

Keywords fluorinated adenosine derivative; pantothenate synthetase; anti-tuberculosis; total synthesis
\end{abstract}

研发高效、低毒的新型抗结核药物引起了人们的广 泛兴趣 ${ }^{[1]}$. 乔春华等 ${ }^{[2]}$ 合成了一系列腺苷类衍生物, 药 理实验显示其具有较强的体外抗结核分支杆菌活性. 目 前, 得益于生命科学的迅猛发展, 尤其是结核菌基因组 全序列的解明 ${ }^{[3]}$, 抗结核菌的新靶位被陆续发现. 其中, 泛酸合成途径 ${ }^{[4]}$ 中泛酸合成酶 $(R v 3602 \mathrm{e}, \mathrm{PanC})$ 作为一个 非常理想的抗结核药物作用靶点开始引起了人们的重 视. 泛酸是合成辅酶 $\mathrm{A}$ 和酰基载体蛋白的必需前体, 是 结核杆菌存活必需的营养物质. 同时, 由于人体所需的 泛酸是直接从食物中摄取的, 人体内不存在泛酸合成途 径, 因此泛酸合成酶作为结核杆菌泛酸合成途径中的一 个关键酶, 成为抗结核药物合适靶位.

泛酸合成酶催化泛解酸 $(\alpha, \gamma$-二羟- $\beta$-二甲基丁酸)与 $\beta$-丙氨酸形成泛酸的反应机理已被阐明 ${ }^{[5]}$ (Scheme 1).
第一步，底物三磷酸腺苷(ATP)和 $\alpha, \gamma$-二羟- $\beta$-二甲基丁 酸结合到酶的活化位点, 首先生成一个与酶紧密结合的 中间体过渡态 $\mathbf{a}$ 一泛酰腺嘌呤核苷酸，同时释放出副 产物焦磷酸 $(\mathrm{PPi})$; 第二步, 另一底物分子 $\beta$-丙氨酸亲核

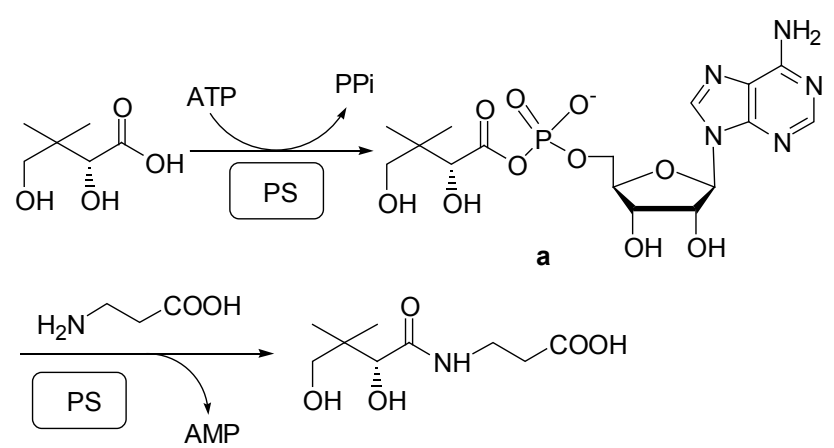

Scheme 1

*E-mail: qiaochunhua@suda.edu.cn

Received December 1, 2012; revised January 26, 2013; published online February 5, 2013.

Project supported by the National Natural Science Foundation of China (No. 81072514) and the National Natural Science Foundation for Young Scientists of China (No. 21002067).

国家自然科学基金(No. 81072514)和国家自然青年科学基金(No. 21002067)资助项目. 
进攻泛酰中间体活泼的羰基碳原子, 生成最终产物泛 酸，同时释放腺苷一磷酸(AMP).

2006 年, Tuck 等 ${ }^{[6]}$ 利用生物电子等排原理设计了一 系列的泛酸合成酶催化反应中间体 $\mathbf{a}$ 的类似物(图 1). 其 中, 化合物 $\mathbf{b}$ (图 1) 对泛酸合成酶的抑制作用最强. 同年, Somu 等 ${ }^{[7]}$ 报道了一系列抗结核载铁蛋白抑制剂的腺苷 类似物, 在对腺苷糖环进行构效关系研究中发现腺苷糖 环 2 位上的取代基对化合物稳定性有一定的影响. 而近 几年来新药研究发现，一些化合物在引入氟原子后具有 更好的生物活性 ${ }^{[8,9]}$, 并且化学稳定性增强 ${ }^{[10]}$. 因此我 们在化合物 $\mathbf{b}$ 的基础上, 用氟取代糖环上 $2^{\prime}$ 位羟基, 提 高其稳定性; 同时, 我们将化合物 $\mathbf{b}$ 羰基 $\alpha$ 位碳从混旋 改为 $R$ 构型, 与反应中间体 $\mathbf{a}$ 的立体构型一致, 设计合 成得到目标化合物 1 .
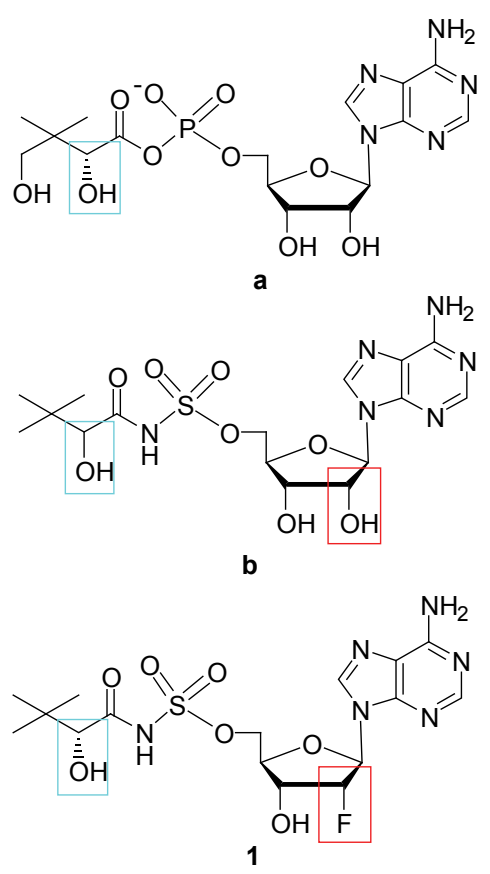

图 1 反应中间体 $\mathbf{a} 、$ 化合物 $\mathbf{b}$ 及目标化合物 $\mathbf{1}$ 的化学结构

Figure 1 Structures of compounds $\mathbf{a}, \mathbf{b}$ and $\mathbf{1}$

\section{1 结果与讨论}

化合物 1 的合成如 Scheme 2 所示: 以 $D$-叔亮氨酸 为原料, 采用文献报道的方法 ${ }^{[11]}$ 将氨基转换为着基得 化合物 2, 该反应中碳原子的手性保持. 化合物 2 经保 护、酯化反应得中间体 $\mathbf{4}$, 三步总产率 $38 \%$ 与文献 $[7,11$, 12]相当; 以阿糖腺苷为原料, 经选择性保护 $3^{\prime}$ 位和 $5^{\prime}$ 位 羟基, 用二乙胺基三氟化硫(DAST)氟代得化合物 6. 化 合物 6 用 $25 \%$ ( $\phi$, 下同)三氟乙酸水溶液脱去 $5^{\prime}$ 位保护 基, 再与氯磺酰胺反应得到中间体 8 ; 中间体 4 与 8 以碳 酸铯为碱反应得到化合物 $\mathbf{9}$, 并在用硅胶柱纯化时加入 $0.3 \%$ 三乙胺使其形成稳定的盐, 最后用 $80 \%$ 三氟乙酸水
溶液脱去 TBS 保护基得到目标产物 $\mathbf{1}$.
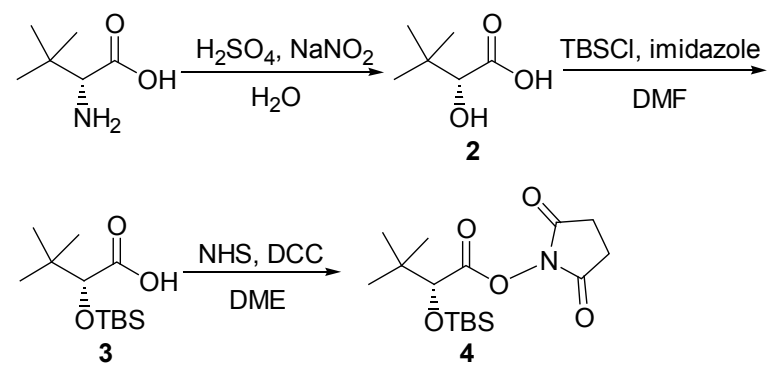

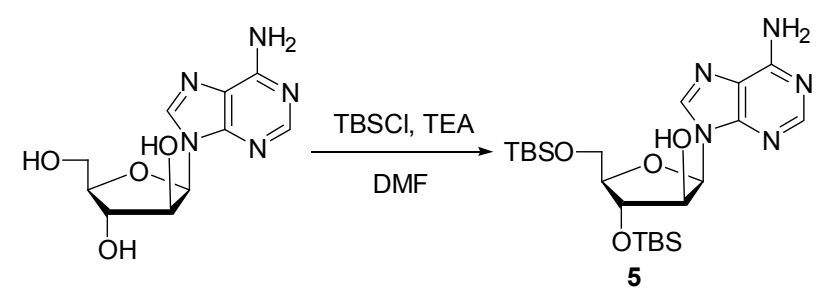

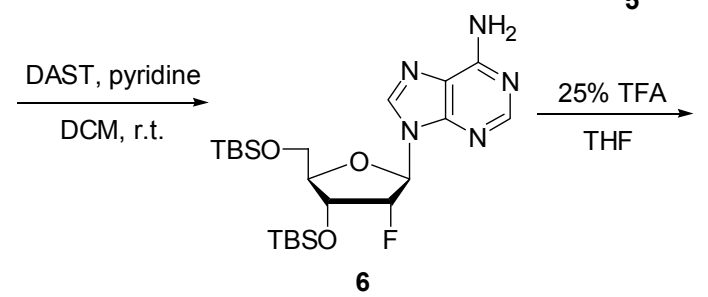

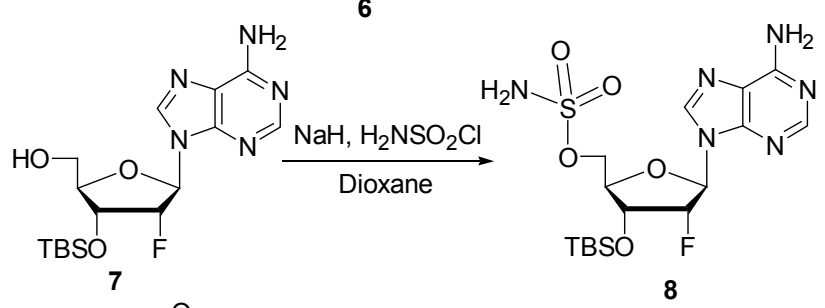

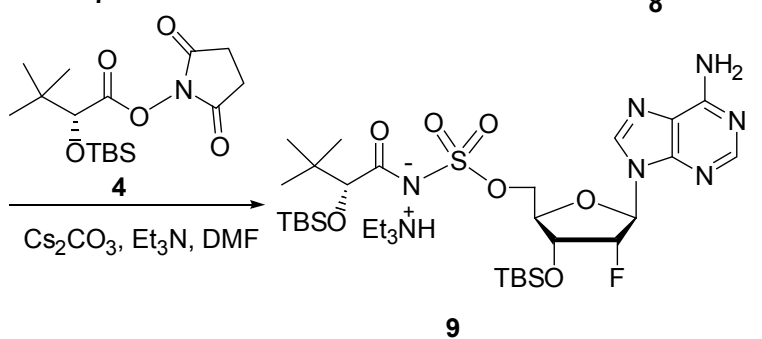

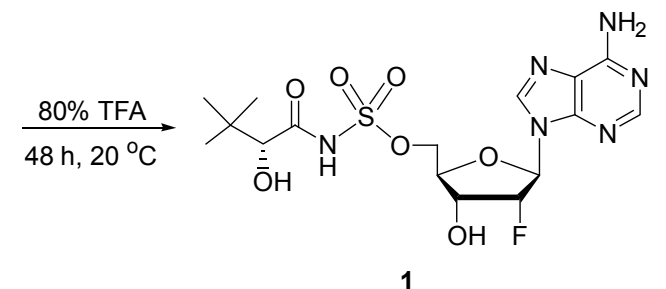

Scheme 2

在 6 的合成中，以阿糖腺苷为原料，由于 $2^{\prime}$ 位羟基 与碱基同在糖环上方，其位阻较大，可以用叔丁二甲基

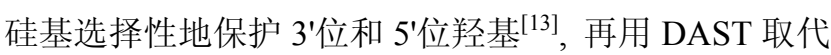
$2^{\prime}$ 位 $S$ 构型羟基得到 $2^{\prime}$ 位 $R$ 构型氟代腺苷中间体 6 , 因氟 代反应杂质较多, 两步总产率为 $20 \%$, 而文献[14]报道 的 2 '位 $R$ 构型氟代腺苷类似物中间体的合成(Scheme 3) 是以腺苷为原料，用三苯甲基选择性保护羟基，经过 4 
步反应，收率只有 $9 \%$. 通过改进合成路线，不仅缩短了 反应步数, 还提高了产率. 化合物 6 未见报道, 但化合 物 7 和 8 的谱图与文献[15]报道一致, 间接证明了化合 物 6 结构正确.

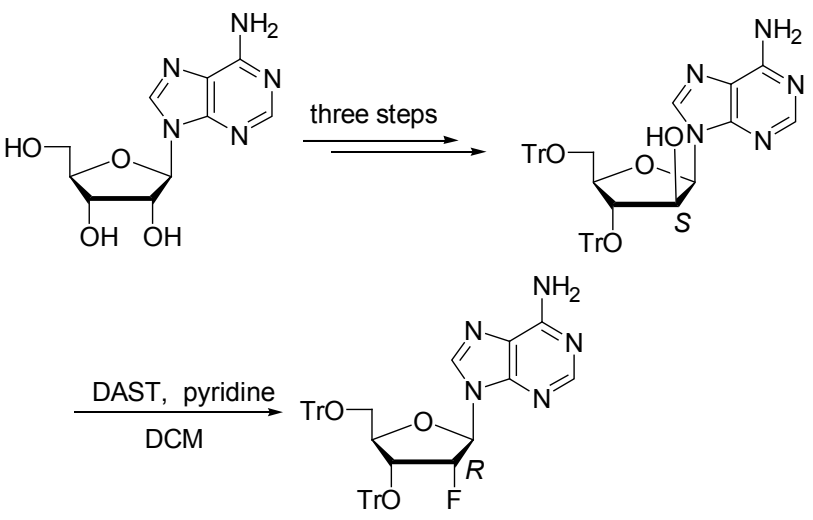

Scheme 3

在 9 的合成中, 用二氯甲烷和甲醇作为洗脱剂硅胶 柱纯化后, 产物会逐渐转变成为极性更大的一些化合 物, 稳定性不足, 我们发现在洗脱剂中加入 $0.3 \%$ 的三乙 胺后, 产物可以稳定存在. 在 $\mathbf{1}$ 的合成中, 分别对反应 温度和三氟乙酸浓度进行了条件优化. 对于温度分别尝 试了在 $0,20,30{ }^{\circ} \mathrm{C}$ 下用 $80 \%$ 三氟乙酸水溶液脱保护, 在 $20{ }^{\circ} \mathrm{C}$ 下脱保护 $48 \mathrm{~h}$, 反应较快, 且 TLC 板观察产物 点附近杂质较少; 对于三氟乙酸浓度分别尝试了在 20 ${ }^{\circ} \mathrm{C}$ 下用 $60 \%, 80 \%, 90 \%$ 三氟乙酸水溶液脱保护, 用 $80 \%$ 三氟乙酸水溶液脱保护 $48 \mathrm{~h}$, TLC 板观察产物点附近的 杂质较少. 最终选择在 $20{ }^{\circ} \mathrm{C}$ 下用 $80 \%$ 三氟乙酸水溶液 脱保护. 最后两步总产率 $6 \%$, 与文献报道的化合物 $\mathbf{b}$ 的 最后两步的产率相当 ${ }^{[6]}$.

\section{2 结论}

通过上述 9 步反应合成得到了一个新的氟代腺苷类 衍生物, 其结构经 ${ }^{1} \mathrm{H} \mathrm{NMR},{ }^{13} \mathrm{C}$ NMR 和 HRMS 表征, 其 与泛酸合成酶的结合常数及对结核菌的抑制活性正在 测定中. 希望得到一个良好的泛酸合成酶抑制剂, 让人 们早日摆脱结核病的困扰.

\section{3 实验部分}

\section{1 仪器与试剂}

UNITY INOVA 型核磁共振仪; 6220 Accurate-Mass TOF LC/MS 液相色谱质谱联用仪; Micromass TOF-MS 型高分辨质谱仪; Autopol III 自动旋光仪; XT5 显微熔点 测定仪.

所用试剂均为市售化学纯或分析纯, 其中乙二醇二 甲醚(DME)、吡啶、二氧六环、 $N, N$-二甲基甲酰胺(DMF)
用前均经除水处理.

\section{2 实验方法}

\subsection{1（R)-2-羟基-3,3-二甲基丁酸(2)的合成}

将 $D$-叔亮氨酸 $(1.0 \mathrm{~g}, 7.62 \mathrm{mmol}, 1.0$ equiv.)溶于 $30.5 \mathrm{~mL} 0.5 \mathrm{~mol} \cdot \mathrm{L}^{-1} \mathrm{H}_{2} \mathrm{SO}_{4}$ 中, 冰浴下, 用自动进样器 向其逐滴滴加 $\mathrm{NaNO}_{2}$ 的水溶液 ( $3.15 \mathrm{~g}$ 溶于 $10 \mathrm{~mL}$ 水中), 滴速 $20 \mathrm{~mL} / \mathrm{h}$, 缓慢升至室温, 摚拌过夜. 加 $30 \mathrm{~mL}$ 水稀 释, 用乙醚 $(30 \mathrm{~mL} \times 3)$ 和水萃取, 有机层用无水硫酸钠 干燥. 有机层旋干, 得 $806 \mathrm{mg}$ 淡黄色油状液体 2 , 产率 $80 \% .[\alpha]_{\mathrm{D}}^{25.1}-4.68\left(c\right.$ 1.11, $\left.\mathrm{H}_{2} \mathrm{O}\right)$ [文献值 ${ }^{[16]}:[\alpha]_{\mathrm{D}}^{25}-$ 4.3 (c 4.0, $\left.\mathrm{H}_{2} \mathrm{O}\right)$ ]; ${ }^{1} \mathrm{H}$ NMR (400 MHz, $\left.\mathrm{CDCl}_{3}\right) \delta: 3.90$ (s, $1 \mathrm{H}, \mathrm{CH}), 1.02\left[\mathrm{~s}, 9 \mathrm{H},\left(\mathrm{CH}_{3}\right)_{3}\right]$.

\subsection{2（R)-2- $O$-叔丁二甲基砝基-3,3-二甲基丁酸(3)的 合成}

将 2 (2.06 g, $15.6 \mathrm{mmol}, 1.0$ equiv.) 溶于 $15 \mathrm{~mL} \mathrm{DMF}$ 中, 加入咪唑 $(5.09 \mathrm{~g}, 74.88 \mathrm{mmol}, 4.8$ equiv.)及叔丁二甲 基硅氯(TBSCl) (5.64 g, $37.44 \mathrm{mmol}, 2.4$ equiv.), 室温摚 拌过夜. 用乙酸乙酯/石油醚 $(V: V=1: 1)$ 和水萃取, 有 机相依次用 $10 \%$ 柠檬酸、水、饱和 $\mathrm{NaHCO}_{3}$ 、饱和食盐 水、水洗涤. 有机层用无水硫酸钠干燥. 旋干, 用 100 $\mathrm{mL}$ 甲醇溶解, 加入 $\mathrm{K}_{2} \mathrm{CO}_{3}$ 水溶液 (5.6 $\mathrm{g} \mathrm{K}_{2} \mathrm{CO}_{3}$ 溶于 45 $\mathrm{mL}$ 水中), 搅拌 $4 \mathrm{~h}$. 旋干甲醇, 用 $10 \%$ 柠檬酸调节 $\mathrm{pH}$ 到 4 , 用乙酸乙酯萃取. 有机层用无水硫酸钠干燥. 旋 干, 硅胶柱分离纯化 $[V$ (石油醚) $: V($ 乙酸乙酯 $)=10 ： 1]$, 合并, 浓缩得 $3.1 \mathrm{~g}$ 油状物 $\mathbf{3}$, 产率 $81 \%$. [ $\alpha]_{\mathrm{D}}^{23.7}+8.1(\mathrm{c}$ $0.16, \mathrm{MeOH}$ ); ${ }^{1} \mathrm{H} \mathrm{NMR}\left(400 \mathrm{MHz}, \mathrm{CDCl}_{3}\right) \delta: 9.64$ (br s, $1 \mathrm{H}, \mathrm{COOH}), 3.83(\mathrm{~s}, 1 \mathrm{H}, \mathrm{CH}), 0.97\left[\mathrm{~s}, 9 \mathrm{H},\left(\mathrm{CH}_{3}\right)_{3}\right], 0.93$ [s, 9H, $\left.\left(\mathrm{CH}_{3}\right)_{3} \mathrm{CSi}\right], 0.08\left(\mathrm{~s}, 3 \mathrm{H}, \mathrm{SiCH}_{3}\right), 0.06(\mathrm{~s}, 3 \mathrm{H}$, $\left.\mathrm{SiCH}_{3}\right) ;{ }^{13} \mathrm{C}$ NMR $\left(400 \mathrm{MHz}, \mathrm{CDCl}_{3}\right) \delta: 176.01,79.95$, 35.42, 26.01, 25.81, 18.26, -5.17; ESI-MS $m / z: 245.2$ $[\mathrm{M}-\mathrm{H}]^{-}$.

3.2.3 (R)-2- $O$-叔丁二甲基硅基-3,3-二甲基丁酸琥珀 酰亚胺酯(4)的合成

将 3 (2.0 g, $8.11 \mathrm{mmol}, 1.0$ equiv.) 溶于 $50 \mathrm{~mL} \mathrm{DME}$ 中, 冰浴下, 加入 NHS (1.87 g, $16.2 \mathrm{mmol}, 2.0$ equiv.)及 环已基碳二亚胺(DCC) (2.34 g, $11.3 \mathrm{mmol}, 1.4$ equiv.), 室温反应 $24 \mathrm{~h}$. 硅藻土过滤, 旋干, 硅胶柱分离纯化 [ $V$ (石油醚) $: V$ (二氯甲烷 $)=1: 1$ ], 合并得 $1.6 \mathrm{~g}$ 油状物 4, 产率 60\%. $[\alpha]_{\mathrm{D}}^{25.1}+12.7$ (c 1.02, MeOH); ${ }^{1} \mathrm{H}$ NMR $\left(400 \mathrm{MHz}, \mathrm{CDCl}_{3}\right) \delta: 4.07(\mathrm{~s}, 1 \mathrm{H}, \mathrm{CH}), 2.80(\mathrm{~s}, 4 \mathrm{H}$, $\left.\mathrm{CH}_{2} \mathrm{CH}_{2}\right), 1.03$ [s, 9H, $\left(\mathrm{CH}_{3}\right)_{3}$ ], 0.90 [s, 9H, $\left(\mathrm{CH}_{3}\right)_{3} \mathrm{CSi}$, 0.10 (s, $3 \mathrm{H}, \mathrm{SiCH}_{3}$ ), 0.07 (s, 3H, $\left.\mathrm{SiCH}_{3}\right) ;{ }^{13} \mathrm{C}$ NMR (400 $\left.\mathrm{MHz}, \mathrm{CDCl}_{3}\right) \delta: 169.07,168.09,78.50,35.97,25.69$, 18.17, - 5.23, - 5.45; ESI-MS m/z: $342.8[\mathrm{M}-\mathrm{H}]^{-}$. 
3.2.4 3',5'-O-二(叔丁二甲基硅基)阿糖腺苷(5)的合 成

将阿糖腺苷(2.0 g, $7.48 \mathrm{mmol}, 1.0$ equiv.)溶于 $40 \mathrm{~mL}$ DMF 中, 加入三乙胺 $(5.2 \mathrm{~mL}, 37.42 \mathrm{mmol}, 5.0$ equiv.), 再加入 TBSCl (2.82 g, $18.71 \mathrm{mmol}, 2.5$ equiv.), 常温下 搅拌过夜, TLC 监测反应完全. 用水和乙酸乙酯(100 $\mathrm{mL} \times 3)$ 萃取, 硅胶柱分离纯化 $[V($ 二氯甲烷 $): V($ 甲 醇 $)=80: 1$ ], 得 $2.4 \mathrm{~g}$ 白色固体 $\mathbf{5}$, 产率为 $65 \%$. m.p. $176 \sim 177{ }^{\circ} \mathrm{C}$ [文献值 ${ }^{[9]}$ : m.p. $176 \sim 178{ }^{\circ} \mathrm{C}$ ]; $[\alpha]_{\mathrm{D}}^{27.1}$ +4.4 (c 1.00, MeOH); ${ }^{1} \mathrm{H}$ NMR $\left(400 \mathrm{MHz}, \mathrm{CDCl}_{3}\right) \delta$ : 8.35 (s, 1H, H-2), 8.27 (s, 1H, H-8), 6.33 (s, 1H, H-1'), 5.64 (br s, $2 \mathrm{H}, \mathrm{NH}_{2}$ ), 4.88 (d, $J=10.1 \mathrm{~Hz}, 1 \mathrm{H}, \mathrm{H}-2$ '), 4.35 (s, 1H, OH), 4.14 (d, J=9.9 Hz, 1H, H-3'), 4.07 (s, 1H, H-4'), 3.96 (d, $\left.J=11.2 \mathrm{~Hz}, 1 \mathrm{H}, \mathrm{CH}_{2}-5^{\prime}\right), 3.81$ (d, $J=11.1$ $\left.\mathrm{Hz}, 1 \mathrm{H}, \mathrm{CH}_{2}-5^{\prime}\right), 0.93\left[\mathrm{~s}, 18 \mathrm{H}, 2 \times\left(\mathrm{CH}_{3}\right)_{3}\right], 0.15$ [d, $J=5.6$ $\left.\mathrm{Hz}, 12 \mathrm{H}, 2 \times \mathrm{Si}\left(\mathrm{CH}_{3}\right)_{2}\right]$; ESI-MS $m / z: 496.4[\mathrm{M}+\mathrm{H}]^{+}$.

3.2 .52 '- 去氧-2'-氟-3',5'-O-二(叔丁二甲基硅基)腺苷 (6)的合成

将吡啶 $(0.2 \mathrm{~mL}, 10.00 \mathrm{mmol}, 10.0$ equiv.)溶于 $2 \mathrm{~mL}$ 干燥二氯甲烷中, 氮气保护下加入 DAST $(0.13 \mathrm{~mL}, 1.00$ mmol, 5.0 equiv.), 再将 5 (100 mg, 0.20 mmol, 1.0 equiv.) 溶于 $1.0 \mathrm{~mL}$ 二氯甲烷后加入到上述溶液中, 常温下摚 拌反应 $6 \mathrm{~h}$. 加入 $1.5 \mathrm{~mL} 5 \%$ 碳酸氢钠溶液淬灭反应, 用 二氯甲烷 $(10 \mathrm{~mL} \times 3)$ 和水萃取, 用蒸馏水洗一次, 硅胶 柱分离纯化 $[V$ (二氯甲烷) : $V$ (甲醇 $)=50: 1$ ], 得 20.1 $\mathrm{mg}$ 白色固体 6, 产率为 $31 \%$. m.p. $148 \sim 150{ }^{\circ} \mathrm{C} ;[\alpha]_{\mathrm{D}}^{25.2}$ +5.8 (c 0.66, MeOH); ${ }^{1} \mathrm{H}$ NMR $\left(400 \mathrm{MHz}, \mathrm{CDCl}_{3}\right) \delta$ : 8.32 (s, 1H, H-2), 8.16 (s, 1H, H- $), 6.25$ (d, $J=15.1 \mathrm{~Hz}$, $\left.1 \mathrm{H}, \mathrm{H}-1^{\prime}\right), 6.05$ (br s, $2 \mathrm{H}, \mathrm{NH}_{2}$ ), 5.31 (d, $J=51.5 \mathrm{~Hz}, 1 \mathrm{H}$, H-2'), 4.68 (d, $\left.J=16.0 \mathrm{~Hz}, 1 \mathrm{H}, \mathrm{H}-3^{\prime}\right), 4.14$ (d, $J=8.2 \mathrm{~Hz}$, $\left.1 \mathrm{H}, \mathrm{H}-4^{\prime}\right), 4.02$ (d, $\left.J=9.5 \mathrm{~Hz}, 1 \mathrm{H}, \mathrm{CH}_{2}-5^{\prime}\right), 3.79$ (d, $J=$ $\left.12.1 \mathrm{~Hz}, 1 \mathrm{H}, \mathrm{CH}_{2}-5^{\prime}\right), 0.93\left[\mathrm{~s}, 9 \mathrm{H}, \mathrm{SiC}\left(\mathrm{CH}_{3}\right)_{3}\right], 0.89[\mathrm{~s}, 9 \mathrm{H}$, $\left.\mathrm{SiC}\left(\mathrm{CH}_{3}\right)_{3}\right], 0.13\left[\mathrm{~d}, J=4.4 \mathrm{~Hz}, 6 \mathrm{H}, \mathrm{Si}\left(\mathrm{CH}_{3}\right)_{2}\right], 0.07$ [d, $\left.J=10.4 \mathrm{~Hz}, 6 \mathrm{H}, \mathrm{Si}\left(\mathrm{CH}_{3}\right)_{2}\right] ;{ }^{13} \mathrm{C}$ NMR $\left(300 \mathrm{MHz}, \mathrm{CDCl}_{3}\right)$ $\delta: 155.83,153.25,149.43,139.25,120.08,92.98$ (d, $J=$ $192.2 \mathrm{~Hz}), 86.90$ (d, $J=32.9 \mathrm{~Hz}), 84.04,69.36$ (d, $J=15.8$ $\mathrm{Hz}), 61.29,26.02,25.77,18.52,18.23,-4.75,-5.34$; ESI-MS $m / z: 498.3[\mathrm{M}+\mathrm{H}]^{+}, 520.3[\mathrm{M}+\mathrm{Na}]^{+}$.

3.2.6 2'-去氧-2'-氟-3'- $O$-叔丁二甲基硅基腺苷(7)的 合成

将 $6(100 \mathrm{mg}, 0.2 \mathrm{mmol})$ 溶于 $0.5 \mathrm{~mL}$ THF 中, 加入 $25 \%$ 三氟乙酸溶液 $2 \mathrm{~mL}$, 常温下搅拌反应 $20 \mathrm{~min}$ 后, 加入饱和碳酸氢钠溶液, 直到无气泡生成. 用乙酸乙酯 $(30 \mathrm{~mL} \times 3)$ 和水萃取, 用饱和食盐水洗一次, 再用无水 硫酸钠干燥, 硅胶柱分离纯化 $[V($ 二氯甲烷 $): V($ 甲醇 $)=$
$50: 1]$, 得 $71 \mathrm{mg}$ 白色固体 7, 产率为 $92 \%$. m.p. 246 $248{ }^{\circ} \mathrm{C} ;[\alpha]_{\mathrm{D}}^{25.7}-25.5$ (c $\left.0.11, \mathrm{MeOH}\right) ;{ }^{1} \mathrm{H}$ NMR $(400$ $\left.\mathrm{MHz}, \mathrm{CD}_{3} \mathrm{OD}\right) \delta: 8.35$ (s, 1H, H-2), 8.17 (s, 1H, H-8), 6.25 (d, $J=19.0 \mathrm{~Hz}, 1 \mathrm{H}, \mathrm{H}-1$ '), 5.49 (dt, $J=52.6,4.2 \mathrm{~Hz}, 1 \mathrm{H}$, H-2'), 4.74 (d, $J=11.2 \mathrm{~Hz}, 1 \mathrm{H}, \mathrm{H}-3^{\prime}$ ), 4.13 (s, 1H, H-4'), $3.88\left(\mathrm{~d}, J=12.4 \mathrm{~Hz}, 1 \mathrm{H}, \mathrm{CH}_{2}-5^{\prime}\right), 3.69$ (d, $J=15.0 \mathrm{~Hz}, 1 \mathrm{H}$, $\left.\mathrm{CH}_{2}-5^{\prime}\right), 0.94$ [s, 9H, $\mathrm{SiC}\left(\mathrm{CH}_{3}\right)_{3}$ ], $0.16[\mathrm{~d}, J=3.6 \mathrm{~Hz}, 6 \mathrm{H}$, $\left.\mathrm{Si}\left(\mathrm{CH}_{3}\right)_{2}\right] ;{ }^{13} \mathrm{C}$ NMR $\left(300 \mathrm{MHz}, \mathrm{DMSO}-d_{6}\right) \delta: 156.20$, 152.62, 148.84, 139.59, 119.17, 92.25 (d, $J=189.7 \mathrm{~Hz})$, 85.69 (d, $J=32.4 \mathrm{~Hz}), 84.71,69.99$ (d, $J=15.0 \mathrm{~Hz}), 60.29$, 25.61, 17.85, -4.99; ESI-MS $m / z: 384.2[\mathrm{M}+\mathrm{H}]^{+}$.

3.2.7 5'- $O$-磺酰胺基-2'-去氧-2'-氟-3'- $O$-叔丁二甲基 硅基腺苷 $(8)$ 的合成

将 7 (100 mg, $0.26 \mathrm{mmol}, 1.0$ equiv.)溶于 $5 \mathrm{~mL}$ 干燥 二氧六环中, 氮气保护, 加入 $\mathrm{NaH}(32 \mathrm{mg}, 60 \%, 0.78$ mmol, 3.0 equiv.), 常温下反应 $1 \mathrm{~h}$ 后, 加入 $\mathrm{NH}_{2} \mathrm{SO}_{2} \mathrm{Cl}$ (75 mg, $0.65 \mathrm{mmol}, 2.5$ equiv.), 常温下反应 $5 \mathrm{~h}$ 后, 用甲 醇淬灭反应, 硅胶柱分离纯化 $[V$ (二氯甲烷 $): V$ (甲醇 $)=$ $30 ： 1$ 得 $72 \mathrm{mg}$ 白色固体 8 , 产率为 $60 \%$. m.p. $>236{ }^{\circ} \mathrm{C}$ (dec.); $[\alpha]_{\mathrm{D}}^{25.2}+2.8$ (c 1.16, MeOH); ${ }^{1} \mathrm{H}$ NMR $(300$ $\left.\mathrm{MHz}, \mathrm{CD}_{3} \mathrm{OD}\right) \delta: 8.27$ (s, $\left.1 \mathrm{H}, \mathrm{H}-2\right), 8.22$ (s, $\left.1 \mathrm{H}, \mathrm{H}-8\right), 6.32$ (dd, $\left.J=17.1,2.6 \mathrm{~Hz}, 1 \mathrm{H}, \mathrm{H}-1^{\prime}\right), 5.55$ (dd, $J=44.8,9.6 \mathrm{~Hz}$, 1H, H-2'), 4.93 (s, 1H, H-4'), 4.44 (d, $J=8.1 \mathrm{~Hz}, 1 \mathrm{H}, \mathrm{H}-3^{\prime}$ ), $4.31\left(\mathrm{~d}, J=4.6 \mathrm{~Hz}, 1 \mathrm{H}, \mathrm{CH}_{2}-5^{\prime}\right), 4.28(\mathrm{~d}, J=3.9 \mathrm{~Hz}, 1 \mathrm{H}$, $\left.\mathrm{CH}_{2}-5^{\prime}\right), 0.97$ [s, 9H, $\left.\mathrm{SiC}\left(\mathrm{CH}_{3}\right)_{3}\right], 0.21[\mathrm{~d}, J=4.1 \mathrm{~Hz}, 6 \mathrm{H}$, $\left.\mathrm{Si}\left(\mathrm{CH}_{3}\right)_{2}\right] ;{ }^{13} \mathrm{C}$ NMR (300 MHz, DMSO- $\left.d_{6}\right) \delta: 156.20$, 152.77, 148.84, 139.73, 119.13, 92.20 (d, $J=188.4 \mathrm{~Hz}$ ), 86.07 (d, $J=33.7 \mathrm{~Hz}), 80.28,70.15$ (d, $J=15.6 \mathrm{~Hz}), 67.70$, 25.62, 17.80, -4.89, -5.09; ESI-MS $m / z$ : $463.2[\mathrm{M}+$ $\mathrm{H}]^{+}$.

3.2.8 5'- $O$-\{[(R)-2-O-叔丁二甲基硅-3,3-二甲基丁酰 基]磺酰胺基\}-2'-去氧-2'-氟-3'- $O$-叔丁二甲基硅基腺 苷三乙胺盐(9)的合成

将 8 (70 mg, $0.15 \mathrm{mmol}, 1.0$ equiv.)溶于 $5 \mathrm{~mL}$ 干燥 DMF 中, 氮气保护下, 加入 $\mathrm{Cs}_{2} \mathrm{CO}_{3}(98 \mathrm{mg}, 0.30 \mathrm{mmol}$, 2.0 equiv.)和 4 (103 mg, $0.30 \mathrm{mmol}, 2.0$ equiv.), 常温下 反应 $20 \mathrm{~h}$. 旋除溶剂, 硅胶柱分离纯化 [ $V$ (二氯甲烷) : $V$ (甲醇) $: V$ (三乙胺) $=500: 6: 1.5$ ), 得 $48 \mathrm{mg}$ 白色固体 9, 产率为 $40 \%$. m.p. $>195{ }^{\circ} \mathrm{C}$ (dec.); $[\alpha]_{\mathrm{D}}^{25.5}-1.3(c$ $0.60, \mathrm{MeOH}) ;{ }^{1} \mathrm{H} \mathrm{NMR}\left(300 \mathrm{MHz}, \mathrm{CDCl}_{3}\right) \delta: 8.33(\mathrm{~s}, 1 \mathrm{H}$, $\mathrm{H}-2$ ), 8.30 (s, $1 \mathrm{H}, \mathrm{H}-8), 6.30$ (dd, $J=14.2,4.0 \mathrm{~Hz}, 1 \mathrm{H}$, H-1'), 5.82 (br s, $2 \mathrm{H}, \mathrm{NH}_{2}$ ), 5.40 (dd, $J=52.4,8.7 \mathrm{~Hz}, 1 \mathrm{H}$, H-2'), 4.70 (d, $\left.J=10.9 \mathrm{~Hz}, 1 \mathrm{H}, \mathrm{H}-3^{\prime}\right), 4.42$ (d, $J=8.5 \mathrm{~Hz}$, $\left.1 \mathrm{H}, \mathrm{H}-4^{\prime}\right), 4.29$ (d, $\left.J=8.6 \mathrm{~Hz}, 2 \mathrm{H}, \mathrm{CH}_{2}-5^{\prime}\right), 3.73[\mathrm{~s}, 1 \mathrm{H}$, $\left(\mathrm{CH}_{3}\right)_{3} \mathrm{CCH}$ ], 3.08 [q, $\left.J=7.3 \mathrm{~Hz}, 6 \mathrm{H},\left(\mathrm{CH}_{3} \mathrm{CH}_{2}\right)_{3} \mathrm{~N}\right], 1.37$ 
[t, $\left.J=7.3 \mathrm{~Hz}, 9 \mathrm{H},\left(\mathrm{CH}_{3} \mathrm{CH}_{2}\right)_{3} \mathrm{~N}\right], 0.93\left[\mathrm{~s}, 9 \mathrm{H},\left(\mathrm{CH}_{3}\right)_{3}\right], 0.92$ [s, 9H, $\left.\left(\mathrm{CH}_{3}\right)_{3}\right], 0.88$ [s, 9H, $\left.\left(\mathrm{CH}_{3}\right)_{3} \mathrm{C}\right], 0.14[\mathrm{~d}, J=2.1 \mathrm{~Hz}$, $\left.6 \mathrm{H}, \mathrm{Si}\left(\mathrm{CH}_{3}\right)_{2}\right], 0.03\left[\mathrm{~d}, J=0.8 \mathrm{~Hz}, 6 \mathrm{H}, \mathrm{Si}\left(\mathrm{CH}_{3}\right)_{2}\right] ;{ }^{13} \mathrm{C}$ NMR $\left(400 \mathrm{MHz}, \mathrm{CDCl}_{3}\right) \delta$ : 179.18, 155.57, 153.01, 149.42, 139.33, 119.34, 92.40 (d, $J=175.1 \mathrm{~Hz}), 85.65$ (d, $J=32.2 \mathrm{~Hz}), 83.04,82.20,70.84(\mathrm{~d}, J=15.0 \mathrm{~Hz}), 66.83$, $45.81,35.06,26.33,25.65,25.47,18.07,17.88,8.59$, 3.95, -4.84; ESI-MS $m / z: 689.2[\mathrm{M}-\mathrm{H}]^{-}$.

3.2.9 5'- $O$ - $\{[(R)-2$ - 羟基-3,3-二甲基丁酰基 $]$ 磺酰胺 基\}-2'-去氧-2'-氟腺苷(1)的合成

将 9 (30 mg, $0.038 \mathrm{mmol}, 1.0$ equiv.)置于 $10 \mathrm{~mL}$ 圆底 烧瓶中, 加入 $1.0 \mathrm{~mL} 80 \%$ 三氟乙酸溶液, $20{ }^{\circ} \mathrm{C}$ 下搅拌 $48 \mathrm{~h}$. 旋干, 硅胶柱分离纯化 $[V$ (二氯甲烷) : $V$ (甲醇 $)=$ $10: 1$ ], 得 $3.0 \mathrm{mg}$ 白色固体 $\mathbf{1}$, 产率为 $15 \%$. m.p. $>231$ ${ }^{\circ} \mathrm{C}$ (dec.); $[\alpha]_{\mathrm{D}}^{26.6}+2.0($ c $0.40, \mathrm{MeOH}) ;{ }^{1} \mathrm{H}$ NMR $(400$ $\left.\mathrm{MHz}, \mathrm{CD}_{3} \mathrm{OD}\right) \delta: 8.56(\mathrm{~s}, 1 \mathrm{H}, \mathrm{H}-2), 8.30(\mathrm{~s}, 1 \mathrm{H}, \mathrm{H}-8), 6.68$ (d, $\left.J=6.7 \mathrm{~Hz}, 1 \mathrm{H}, \mathrm{H}-1^{\prime}\right), 5.00$ (dt, $\left.J=30.1 \mathrm{~Hz}, 1 \mathrm{H}, \mathrm{H}-2^{\prime}\right)$, 4.90 (s, 1H, H-3'), 4.84 (s, 1H, H-4'), 4.76 (dd, $J=14.2,2.6$ $\left.\mathrm{Hz}, 1 \mathrm{H}, \mathrm{CH}_{2}-5^{\prime}\right), 4.50$ (dd, $J=18.9,4.5 \mathrm{~Hz}, 1 \mathrm{H}, \mathrm{CH}_{2}-5^{\prime}$ ), $3.63\left[\mathrm{~s}, 1 \mathrm{H},\left(\mathrm{CH}_{3}\right)_{3} \mathrm{CCH}\right], 0.98\left[\mathrm{~s}, 9 \mathrm{H},\left(\mathrm{CH}_{3}\right)_{3} \mathrm{C}\right] ;{ }^{13} \mathrm{C} \mathrm{NMR}$ $\left(400 \mathrm{MHz}, \mathrm{CD}_{3} \mathrm{OD}\right) \delta: 179.05,158.72,150.36,141.00$, 140.52, 121.25, 94.30 (d, $J=193.9 \mathrm{~Hz}), 92.38$ (d, $J=31.4$ $\mathrm{Hz}), 84.79,80.23$ (d, $J=7.8 \mathrm{~Hz}), 71.50(\mathrm{~d}, J=15.9 \mathrm{~Hz})$, 59.49, 36.17, 26.51; HRMS (ESI) calcd for $\mathrm{C}_{16} \mathrm{H}_{23} \mathrm{FN}_{6} \mathrm{O}_{7} \mathrm{~S}$ $[\mathrm{M}-\mathrm{H}]^{-}:$:461.1260, found 461.1281.

\section{References}

[1] (a) Miller, M. J.; Walz, A. J.; Zhu, H. L.; Wu, C. R.; Moraski, G.; Mollmann, U.; Tristani, E. M.; Crumbliss, A. L.; Ferding, M. T.; Checkley, L.; Edwards, R. L.; Boshoff, H. I. J. Am. Chem. Soc. 2011, 133, 2076.

(b) Zhang, X. L.; Hu, Y. W.; Chen, S. D.; Luo, R. S.; Yue, J.; Zhang, Y.; Duan, W. H.; Wang, H. H. Bioorg. Med. Chem. Lett. 2009, 19, 6074.

[2] (a) Qiao, C. H.; Gupte, A.; Boshoff, H. I.; Wilson, D. J.; Bennett, E. M.; Somu, R. V.; Barry, C. E.; Aldrich, C. C. J. Med. Chem. 2007,
50,6080 .

(b) Li, J.; Ao, G. Z.; Chen, J. L.; Qiao, C. H. Chin. J. Synth. Chem. 2009, 17, 696 (in Chinese).

(李静，敖桂珍，陈晶否，乔春华，合成化学, 2009, 17, 696.)

[3] Cole, S. T.; Brosch, R.; Parkhill, J.; Garnier, T.; Churcher, C.; Harris, D.; Gordon, S. V.; Eiglmeier, K.; Gas, S.; Barry, C. E.; Tekaia, F.; Badcook, K.; Basham, D.; Brown, D.; Chillingworth, T.; Connor, R.; Davies, R.; Devlin, K.; Feltwell, T.; Gentles, S.; Hamlin, N.; Holroyd, S.; Hornsby, T.; Jagels, K.; Krogh, A.; Mclean, J.; Moule, S.; Murphy, L.; Oliver, K.; Osborne, J.; Quail, M. A.; Rajandream, M. A.; Rogers, J.; Rutter, S.; Seeger, K.; Skelton, J.; Squares, R.; Squares, S.; Sulston, J. E.; Taylor, K.; Whitehead, S.; Barrell, B. G. Nature 1988, 393, 537.

[4] (a) Mcintosh, E. N.; Purko, M.; Wood, W. A. J. Biol. Chem. 1957, $228,449$.

(b) Yang, Y. H.; Xiao, C. L. Chem. Life 2008, 28, 448 (in Chinese). (杨延辉, 肖春玲, 生命的化学, 2008, 28, 448.)

[5] Webb, M. E.; Smith, A. G.; Abell, C. Nat. Prod. Rep. 2004, 21, 695.

[6] Tuck, K. L.; Saldanha, S. A.; Birch, L. M.; Smith, A. G.; Abell, C. Org. Biomol. Chem. 2006, 4, 3598.

[7] Somu, R. V.; Wilson, D. J.; Bennett, E. M.; Boshoff, H. I.; Celia, L.; Beck, B. J.; Barry, C. E.; Aldrich, C. C. J. Med. Chem. 2006, 49, 7623.

[8] Yan, Z. H.; Lai, K. M.; Tian, W. S.; Yu, Z. X. Acta Chim. Sinica 2012, 70, 1322 (in Chinese). (严兆华, 赖焜民, 田伟生, 余章昕, 化学学报, 2012, 70, 1322.)

[9] Yang, X. Y.; Lü, T. M.; Zhu, S. J.; Wu, F. H. Acta Chim. Sinica 2011, 69, 815 (in Chinese) (杨雪艳, 吕铁梅, 朱胜杰, 吴范宏, 化学学报, 2011, 69, 815.)

[10] Kumaki, Y.; Day, C. W.; Smee, D. F.; Morrey, J. D.; Barnard, D. L. Antiviral Res. 2011, 92, 329.

[11] Franz, N.; Menin, L.; Klok, H. A. Eur. J. Org. Chem. 2009, 31, 5390.

[12] Hamada, Y.; Kondo, Y.; Shibata, M.; Shioiri, T. J. Am. Chem. Soc. 1989, 111, 669

[13] Baker, D. C.; Kumar, S. D.; Waites, W. J.; Arnett, G.; Shannon, W. M.; Higuchi, W. I.; Lambert, W. J. J. Med. Chem. 1984, 27, 270.

[14] Pankiewicz, K. W.; Krzeminski, J.; Ciszewski, L. A.; Ren, W. Y.; Watanabe, K. A. J. Org. Chem. 1992, 57, 553.

[15] Vijver, P. V.; Ostrowski, T.; Sproat, B.; Goebels, J.; Rutgeerts, O.; Aerschot, A. V.; Wear, M.; Herdewijin, P. J. Med. Chem. 2008, 51, 3020 .

[16] Kontos, Z.; Huszthy, P.; Bradshaw, J. S.; Izatt, R. Tetrahedron: Asymmetry 1999, 10, 2087. 